\title{
The Notch coactivator, MAML1, functions as a novel coactivator for MEF2C-mediated transcription and is required for normal myogenesis
}

\author{
Huangxuan Shen, ${ }^{1}$ Abigail S. McElhinny, ${ }^{2}$ Yang Cao, ${ }^{1}$ Ping Gao, ${ }^{1}$ Jingxuan Liu, ${ }^{1}$ Roderick Bronson, ${ }^{3}$ \\ James D. Griffin, ${ }^{1}$ and Lizi $\mathrm{Wu}^{1,4}$ \\ ${ }^{1}$ Department of Medical Oncology, Dana-Farber Cancer Institute and Departments of Medicine, Brigham and Women's \\ Hospital and Harvard Medical School, Boston, Massachusetts 02115, USA; ${ }^{2}$ Department of Cell Biology and Anatomy, \\ University of Arizona, Tucson, Arizona 85724, USA; ${ }^{3}$ Rodent Histopathology Core, Harvard Medical School, \\ Boston, Massachusetts 02115, USA
}

The MAML (mastermind-like) proteins are a family of three cotranscriptional regulators that are essential for Notch signaling, a pathway critical for cell fate determination. Though the functions of MAML proteins in normal development remain unresolved, their distinct tissue distributions and differential activities in cooperating with various Notch receptors suggest that they have unique roles. Here we show that mice with a targeted disruption of the Maml1 gene have severe muscular dystrophy. In vitro, Maml1-null embryonic fibroblasts failed to undergo MyoD-induced myogenic differentiation, further suggesting that Maml1 is required for muscle development. Interestingly, overexpression of MAML1 in C2C12 cells dramatically enhanced myotube formation and increased the expression of muscle-specific genes, while RNA interference (RNAi)-mediated MAML1 knockdown abrogated differentiation. Moreover, we determined that MAML1 interacts with MEF2C (myocyte enhancer factor 2C), functioning as its potent cotranscriptional regulator. Surprisingly, however, MAML1's promyogenic effects were completely blocked upon activation of Notch signaling, which was associated with recruitment of MAML1 away from MEF2C to the Notch transcriptional complex. Our study thus reveals novel and nonredundant functions for MAML1: It acts as a coactivator for MEF2C transcription and is essential for proper muscle development. Mechanistically, MAML1 appears to mediate cross-talk between Notch and MEF2 to influence myogenic differentiation.

[Keywords: MAML1; MEF2C; Notch; muscular dystrophy; myogenesis; transcriptional coactivator]

Supplemental material is available at http://www.genesdev.org.

Received October 12, 2005; revised version accepted January 18, 2006.

Myogenesis is a carefully orchestrated process that is essential not only for muscle development, but also for the regeneration of old and injured muscle fibers. The myogenic program is initiated when mononucleated muscle progenitor cells (myoblasts) expand and exit the cell cycle in response to specific extrinsic signals. The myoblasts fuse, elongate, and develop into multinucleated myotubes, which form mature skeletal muscle. Biochemically, myogenic differentiation is characterized by the expression of muscle-specific genes including desmin, creatine kinase, and muscle myosin (Bailey et al. 2001; Parker et al. 2003).

Two distinct classes of transcription factors regulate

\footnotetext{
${ }^{4}$ Corresponding author.
}

E-MAIL lizi wu@dfci.harvard.edu; FAX (617) 632-4388.

Article published online ahead of print. Article and publication date are at http://www.genesdev.org/cgi/doi/10.1101/gad.1383706. the myogenic expression program, myogenic regulatory factors (MRFs) and myocyte enhancer factor 2 proteins (MEF2) (Black and Olson 1998; Berkes and Tapscott 2005). MRFs are a family of basic helix-loop-helix (bHLH) transcription factors up-regulated in response to myogenic signals and include MyoD, myogenin, Myf5, and MRF4. They form heterodimers with E proteins such as E12 or E47, and bind to specific DNA sites called E-boxes found within many muscle-specific gene promoters. The MRFs have myogenic activities and are able to convert nonmyogenic, mesenchymal cells into myoblasts. On the other hand, the MEF2 proteins are expressed in many different tissues and belong to the MADS box family that includes MEF2A, MEF2B, MEF2C, and MEF2D. The MEF2 family shares conserved MADS and adjacent MEF2 domains that mediate DNA binding and dimerization. They bind as homodimers or 
heterodimers with the MRFs to the AT-rich consensus sequences that are often associated with regions of muscle-specific gene control. Although MEF2 proteins themselves are not myogenic, they interact with MRFs to potentiate MRF-mediated myogenic activities (Molkentin et al. 1995).

The exact molecular mechanisms dictating myogenesis remain unclear. However, it is well established that this process involves multiple complex signaling pathways that modulate both MRF and MEF2 functions (Dias et al. 1994; McKinsey et al. 2001; Lazaro et al. 2002; Liu et al. 2004). One signaling pathway with an emerging and seemingly critical role in muscle stem cell activation and myogenesis is the Notch pathway: an evolutionarily conserved mechanism in which cell-cell interactions influence distinct cellular fates (Artavanis-Tsakonas et al. 1999; Luo et al. 2005). The four mammalian Notch receptors (Notch1-Notch4) are transmembrane proteins that are activated by ligands expressed on the surfaces of neighboring cells. Upon activation, the receptors undergo proteolytic processing events, resulting in the release of the intracellular domain of Notch (ICN). The ICN then translocates to the nucleus and activates the CSL family of DNA-binding transcription factors. Importantly, the cellular effects and target genes of the Notch pathway are highly cell-context dependent. The target genes of Notch include the well-studied bHLH HES gene family.

Notch signaling is involved in pattern formation and cyclic gene expression during somitogenesis, indicating its complicated and tightly regulated role in muscle development (Weinmaster and Kintner 2003; Rida et al. 2004). During postnatal regeneration of skeletal muscle, Notch signaling appears to play distinct roles at different stages. For instance, aged muscles have an impaired ability to produce sufficient myoblasts necessary for regeneration due to the decreased induction of the Notch ligand Delta following injury (Conboy et al. 2003). Forced activation of Notch restored regenerative potentials, suggesting that Notch signaling is required for muscle stem cell activation and proliferation. In contrast, several studies have determined that the Notch pathway must be inhibited for subsequent myoblast fusion and muscle differentiation events (Kopan et al. 1994; Lindsell et al. 1995; Conboy and Rando 2002). Notch signaling is thought to inhibit myogenesis because it activates the transcription of target genes including HES1, which, in turn, inhibit the expression and activities of MyoD. This process requires the transcription factor for the Notch pathway, CSL. Interestingly, however, some aspects of Notch's inhibitory effect on myogenesis appear to be mediated by CSL-independent mechanisms (Shawber et al. 1996; Nofziger et al. 1999). For example, a mutant Notch receptor that is unable to bind to CSL and activate transcription still prevents C2C12 myoblast differentiation. One potential mechanism for the CSL-independent Notch activity is the finding that Notch ICN1 may interact directly with the muscle transcription factor MEF2C, thereby inhibiting MEF2C's transcriptional activity (Wilson-Rawls et al.
1999). Therefore, Notch-mediated inhibition of myogenesis is both CSL dependent and independent, and Notch signaling must be tightly controlled during different myogenic stages.

Previously, three homologous mammalian mastermind-like (MAML) proteins were discovered as transcriptional coactivators for Notch receptors: They form ternary complexes with Notch ICN and CSL in the nucleus and are essential for Notch-mediated transcription of target genes (Lin et al. 2002; Wu et al. 2002). Strikingly, the three MAML members exhibit distinct expression patterns and also cooperate differentially with various Notch receptors in the activation of target genes. Thus, it appears that the MAML family members are not functionally redundant and may contribute to the distinct biological effects of the Notch pathway that occur in different tissues. Recently, MAML family members were implicated in tumorigenesis (Tonon et al. 2003). However, their roles in normal developmental processes, including myogenesis, are unknown. It also remains unclear whether MAML family members function independently of Notch receptors.

In this study, we generated a mouse strain deficient in the Maml1 gene. Maml1 knockout (KO) mice died within the perinatal period, and histological analyses indicated that they exhibit muscular dystrophy. Indeed, their embryonic fibroblasts are unable to undergo myogenic events when transduced with MyoD, and further studies in $\mathrm{C} 2 \mathrm{C} 12$ cells revealed Maml1's critical role in myogenesis. Mechanistically, MAML1 functions as a novel coregulator for MEF2C during myogenesis, but can be recruited away to act as a coregulator for Notch upon activation of the Notch pathway.

\section{Results}

Knockout of the Mamll gene in mice results in muscular dystrophy and perinatal lethality

Previously, we cloned the murine Maml1 gene and characterized its early developmental expression pattern via whole-mount in situ hybridization studies (Wu et al. 2004). Maml1 gene expression is temporally and spatially dynamic and detectable in many tissues. Relatively high expression levels were detected in the developing brain, spinal cords, lung, limb buds, and somites. Previous Northern blotting also revealed that Maml1 is expressed in many adult tissues including skeletal muscle (Wu et al. 2000). To determine the in vivo function of this gene, we generated a targeting vector to delete the first exon and the $5^{\prime}$ promoter region as a gene inactivation strategy (Fig. 1A). The inactivation of the Maml1 gene and consequent loss of the expression of the mRNA transcript and protein was verified by Southern blot analysis (Fig. 1B), Northern blot analysis (Fig. 1C), and Western blot analysis (Fig. 1D), respectively. Mice heterozygous for the Maml1 gene exhibited no discernible phenotype in comparison with wild-type mice. However, mice with the homozygous mutation, $\left(\mathrm{Maml1}^{-1-}\right)$, were delayed in their growth at birth, re- 
A

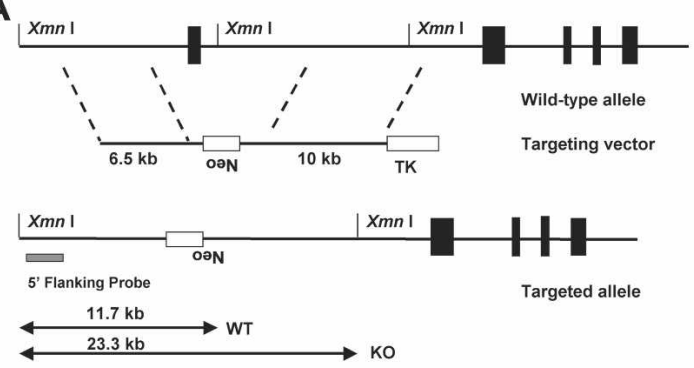

D

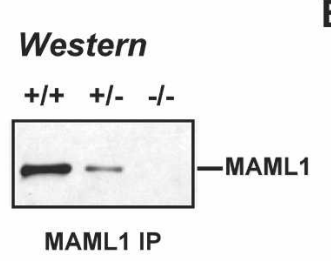

E

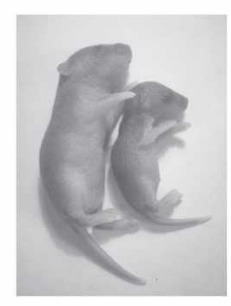

B

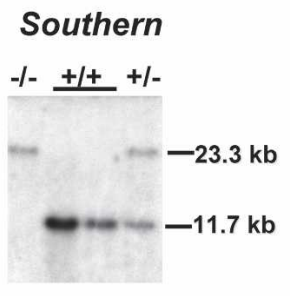

$\mathbf{F}$

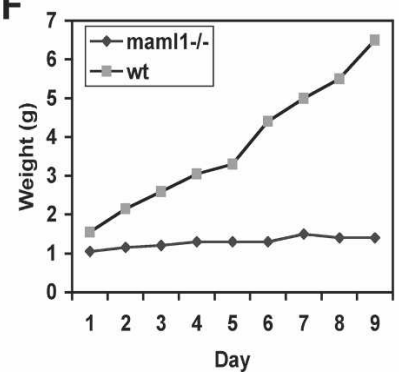

Figure 1. Generation of Maml1-null mice reveals that it is required for normal development. (A) Diagram of the Maml1 targeting construct. Black boxes denote exons of the mouse Maml1 gene. Exon 1 was replaced with a neo gene in the indicated targeting vector. When genomic DNA is digested with XmnI, it is predicted that, using the denoted $5^{\prime}$-flanking probe, 11.7- and 23.3-kb bands would be detected for the wild-type (WT) and KO alleles, respectively. (B) Southern blotting indicated successful targeting of the Maml1 gene. Genomic DNA was isolated from embryos, digested with XmnI, blotted, and hybridized with the $5^{\prime}$-flanking probe. The predicted 23.3-kb band was detected in both the heterozygous and homozygous KO progeny, while the wild type and hetertozygotes contained the 11.7-kb fragment, as expected. (C) Northern blotting showed a loss of Maml1 transcripts in embryonic fibroblasts derived from a Maml1 KO embryo. Note that heterozygotes have reduced levels of Maml1 transcripts compared with wild-type transcripts. The size of the Maml1 transcript is $\sim 6 \mathrm{~kb}$. Relative loading is indicated by 28S rRNA. (D) Western blotting confirmed a loss of MAML1 protein in embryonic fibroblasts derived from a Maml1 KO embryo and a reduction of the protein in the heterozygotes. MAML1 expression $(\sim 140 \mathrm{kDa})$ was analyzed by immunoprecipitation with anti-MAML1 antibodies, followed by Western blotting. (E) Maml1 KO neonate (right) is growth retarded, as compared with its wild-type littermates (left), and die soon after birth (within $10 \mathrm{~d})$. Mice are shown at P5. (F) A representative growth curve reveals that Mam11 KO neonates (black diamonds) fail to thrive, compared with wild-type (WT) littermates (gray squares). Data is presented as weight in grams at successive days after birth.

mained significantly smaller than control mice in the perinatal period (Fig. 1E,F), and died within $\sim 10 \mathrm{~d}$ of birth.

We performed histological analyses on hematoxylin and eosin $(\mathrm{H} \& \mathrm{E})$-stained paraffin sections from both control (wild-type or heterozygous) (Fig. 2A, panels 1,3 ) and homozygous null littermates (Fig. 2A, panels 2,4,5). The most striking observation was that ablation of the Maml1 gene resulted in severe perturbations in skeletal muscle structure. Muscle sections from the $\mathrm{Maml1}^{-/-}$ mice exhibited pathology consistent with muscular dystrophy, including variable myofiber size (both atrophic and hypertrophic), with myofibrillar degeneration and necrosis/loss. In addition, many muscle sections (particularly from the appendicular skeletal muscles) contained a significant number of vacuoles, also consistent with muscle degeneration (Fig. 2A, panels 4,5). This phenotype was mosaic (i.e., some fibers from the null mice appeared normal, while others were severely affected), but the penetrance was $100 \%$. The skeletal muscle lesions indicate that the Maml1 gene is critical for proper muscle development.
Mamll deficiency blocks MyoD-stimulated myogenic conversion in murine embryonic fibroblasts (MEFs), while exogenous MAML1 expression rescues this defect

To investigate the molecular mechanisms by which Maml1 affects myogensis, we undertook a series of cellular and biochemical studies. First, MEFs were generated from wild-type and Maml1 KO littermates. Such cells are known to be capable of differentiating into a variety of cell types. For example, ectopic MyoD expression converts MEFs into cells that express muscle-specific genes and have a distinct myotube morphology (Novitch et al. 1996). We infected self-immortalized MEFs from wild-type and Maml1 KO littermate embryos (embryonic day 12.5 [E12.5]) with a retroviral construct encoding MyoD. After puromycin selection, cells were induced to differentiate. We found that the majority of wild-type MEFs infected with MyoD exhibited differentiation into myotubes, while the MyoD-transduced Mam11 KO MEFs remained in typical fibroblast morphology (Fig. 2B). Moreover, expression of the muscle 
Shen et al.

A
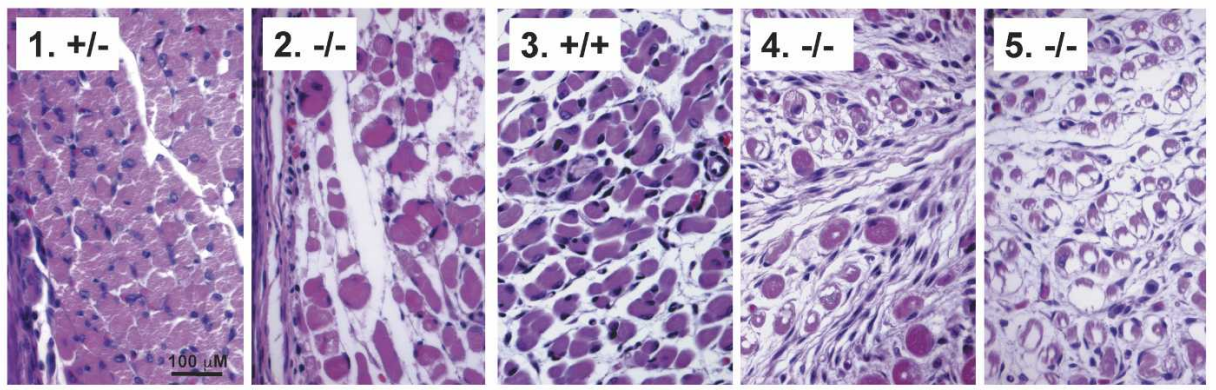

B
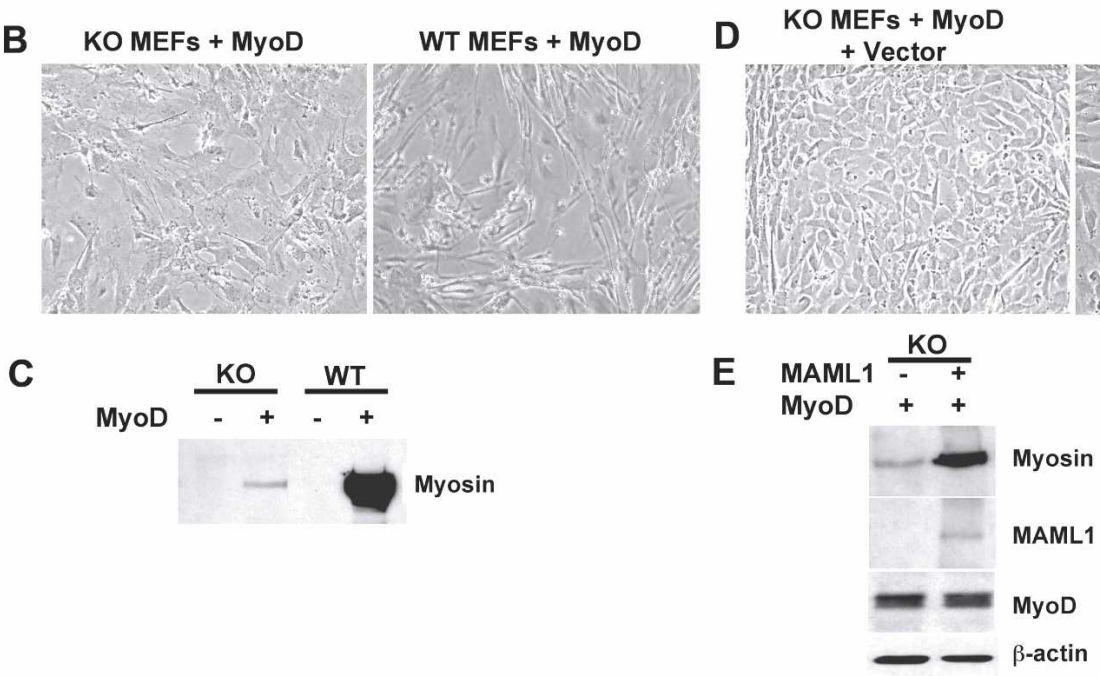

Figure 2. Maml1 deficiency results in muscular dystrophy and defects in MyoD-induced myogenic conversion in MEFs that is rescued upon exogenous Maml1 expression. (A) Whole neonatal mice were sectioned and stained with H\&E. (Panel 1) Hip muscles of a P6 heterozygous mouse shows normal fiber size and distribution. No differences were detected between heterozygotes and wild-type muscles (data not shown). (Panel 2) Hip muscles from a P6 Maml1 KO littermate have marked variation in muscle fiber size and the distribution of fibers is atypical. (Panel 3) Shoulder muscles of a P1 wild-type mouse show normal fiber size and distribution. (Panels 4,5) Advanced dystrophic phenotype in the shoulder muscles of a P1 Maml1 KO littermate showing many hypertrophic and atrophic muscle fibers, and, notably, sarcoplasmic vacuolization. The KO muscles also exhibit a noticeable increase in connective tissue. $(B)$ Maml1 deficiency led to defects in MyoD-induced myogenic convesion in MEFs. Wild-type (right) and Maml1 KO (left) MEFs were infected with MyoD and cultured under differentiation medium for $3 \mathrm{~d}$. (C) Wild-type (WT) MEFs have higher levels of myosin expression than the Maml1 KO MEFs. (- and +) Cells infected with retroviral vectors or viruses expressing MyoD. $(D)$ Exogenous expression of MAML1 in KO MEFs rescues MyoD-induced myotube formation. Stably transduced MyoD KO MEF cells were infected with Flag-tagged MAML1 viruses or controls, and cells were induced for differentiation. (E) Expression of exogenous MAML1, MyoD, and Myosin were determined by Western blot analysis.

marker, myosin was significantly less in MyoD-transduced Maml1 KO MEFs, compared with wild-type MEFs infected with MyoD that robustly expressed myosin (Fig. 2C). These data suggest that Maml1 KO mice are defective in myogenic events regulated by $\mathrm{MyoD}$, and so we next tested whether the phenotype could be rescued by exogenous MAML1 expression. Indeed, upon reintroduction of MAML1 expression in the MyoD-transduced Maml1 KO MEFs, the cells exhibited a significantly augmented degree of myogenic conversion, as indicated by an increased number of myotube-like cells (Fig. 2D). Furthermore, the rescued cells had significantly higher expression levels of myosin (Fig. 2E) compared with the MEFs infected with MyoD, but lacking exogenous MAML1 expression. These data indicate that Maml1 indeed is directly essential for MyoD-induced myogenic events.
Maml1 is expressed throughout C2C12 myoblast differentiation and its overexpression enhances myogenesis

We next performed experiments using the murine myoblast $\mathrm{C} 2 \mathrm{C} 12$ cell line. These cells provide a valuable model for studying myogenic differentiation because, when cultured under conditions to promote differentiation, they fuse, express muscle-specific proteins, and form mature myotubes. First, we analyzed the expression of the Maml1 gene in proliferating and differentiating $\mathrm{C} 2 \mathrm{C} 12$ cells by Western blotting. Our data indicate that the MAML1 protein is expressed at similar levels during the myoblast-to-myotube transition (Supplementary Fig. S1). Thus, Maml1 is expressed throughout C2C12 myoblast differentiation.

We next determined whether ectopic expression of the 
MAML1 gene affected myoblast differentiation. C2C12 cells were infected with MAML1 viruses (pLXSN based) or vector control viruses (see Fig. $3 \mathrm{~A}$ for schematic of the viral constructs used in these studies). After G418 selection, the expression of exogenous MAML1 was confirmed by Western blot analysis (Fig. 3B, lane 2 is fulllength [FL] MAML1). The cells were induced to differentiate, and myotube formation and muscle-specific gene expression were examined. As shown in Figure 3C (panel 2), ectopic MAML1 expression led to the formation of strikingly large, robust myotubes, compared with cells infected with control virus (panel 1). Consistent with these data, MAML1-infected myotubes exhibited enhanced expression of muscle myosin within 2-6 d of differentiation, while MyoD and myogenin levels appeared unaffected (Fig. 3D, cf. lanes 2 and 1).

The results prompted us to ask whether MAML1 enhances myogenesis by regulating muscle gene expression. Thus, we examined the effect of MAML1 expression on the promoter activity of another muscle-specific gene, muscle creatine kinase (MCK). C2C12 cells were transfected with a MCK promoter reporter in the presence of increasing amounts of the MAML1 expression vector. We found that MAML1 expression activated the MCK promoter reporter in a dose-dependent fashion (Fig. $3 \mathrm{E})$. These data suggest that MAML1 is involved in regulation of myogenesis by enhancing muscle gene expression, including myosin and MCK.

To determine the specific domain of the MAML1 protein that is involved in the enhancement of myogenesis, we also generated stably transduced C2C12 cells expressing two previously characterized truncated MAML1 mutants (Fig. 3B, lanes 3,4). Both mutants, MAML1(124-1016) (construct 3, defective in Notch binding) and MAML1(1-302) (construct 4, defective in transcriptional activation), exert dominant-negative effects on Notch signaling (Wu et al. 2000). We found that C2C12 cells expressing MAML1(124-1016) showed substantially reduced myotube formation (Fig. 3C, panel 3) and myosin expression (Fig. 3D, lanes 3), while cells expressing MAML1(1-302) did not have apparent effects (Fig. 3C [panel 4], D [lanes 4]). In addition, MAML1(124-
A

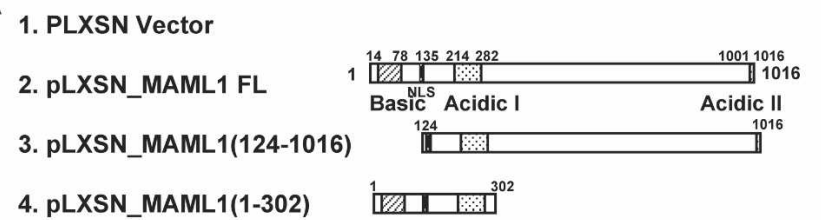

B

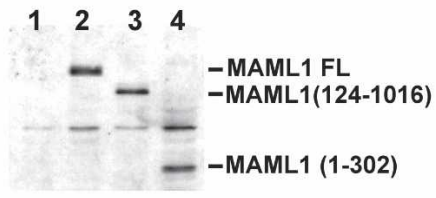

C

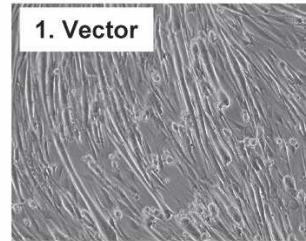

D
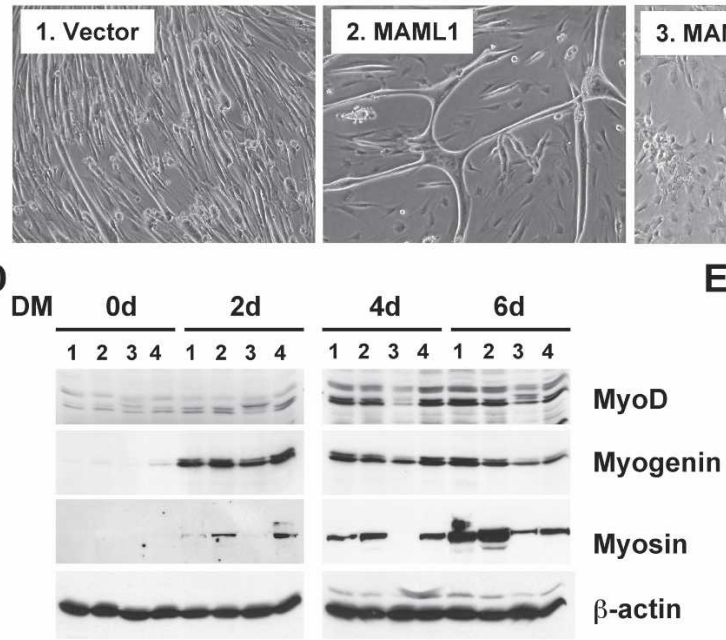
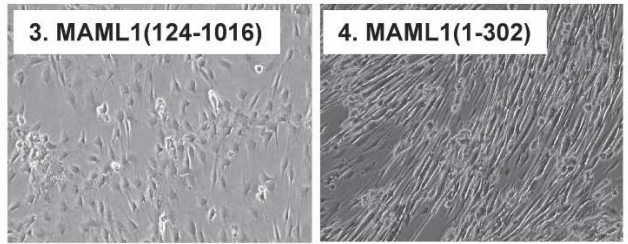

$E$

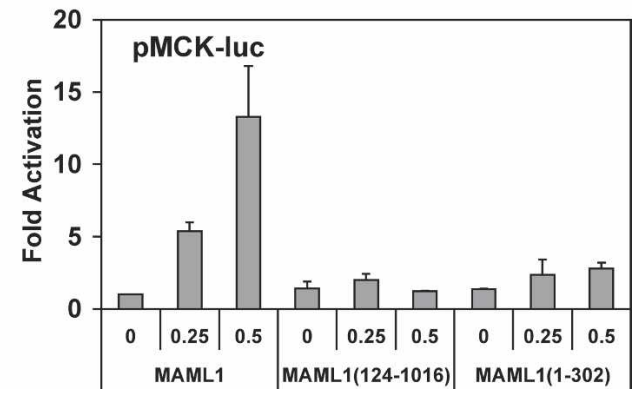

Figure 3. Exogenous MAML1 expression enhances myogenic differentiation of $\mathrm{C} 2 \mathrm{C} 12$ cells, and its $\mathrm{N}$-terminal region is required for this effect. (A) Schematic diagram of FL and truncated MAML1 constructs in the pLXSN viral vectors used in this study. (B) Expression of exogenous MAML1 proteins in C2C12 cells. C2C12 cells were infected with pLXSN-based MAML1 viruses, and selected with G418. Expression of MAML1 proteins was confirmed by blotting with an anti-HA antibody. (C) Overexpression of MAML1 in $\mathrm{C} 2 \mathrm{C} 12$ cells dramatically increases myotube formation and the $\mathrm{N}$-terminal region of MAML1 is required for myogenic enhancement. Stably infected C2C12 Cells were induced to differentiate, and photographed at day 6. (D) Overexpression of MAML1 enhances myosin expression during myogenesis. Whole-cell lysates from infected C2C12 cells were prepared at days 0, 2, 4, and 6 after culturing in DM, and expression levels of muscle proteins were analyzed by Western blot analyses. (E) Expression of MAML1 enhances the activation of the promoter driving MCK. C2C12 cells were transfected with $10 \mathrm{ng}$ of Renilla luciferase plasmid, $0.5 \mu \mathrm{g}$ of pMCK-luc, and increasing amounts of expression plasmids encoding Flag-tagged MAML1. After $24 \mathrm{~h}$ transfection, cells were cultured in DM overnight and lysates were harvested. MCK reporter firefly luciferase activity, corrected for Renilla luciferase, is expressed as fold activation relative to cells not expressing MAML1. 
1016) appeared to inhibit MyoD and myogenin induction (Fig. 3D, lanes 3). Consistent with these data, the two truncated mutants did not significantly activate the MCK promoter compared with FL MAML1 (Fig. 3E). Taken together, these data indicate that MAML1 enhances myogenesis; specifically, it promotes myotube formation and enhances muscle-specific gene expression, including myosin and MCK. Furthermore, the Nterminal region of MAML1, a domain previously found to interact with Notch, is essential for these effects.

\section{RNA interference (RNAi)-mediated knockdown of Mamll expression inhibits muscle cell differentiation}

To determine whether Maml1 is required for myoblast differentiation, we tested several small interfering RNA (siRNA) targeted to different regions of the mouse Maml1 gene, and found that two independent siRNA duplexes, V and VII, significantly knocked down endogenous MAML1 expression. C2C12 cells were transfected with these two Maml1-targeted or control siRNA duplexes, or water-only controls, and cultured under conditions to promote differentiation $48 \mathrm{~h}$ after transfection. Lysates were harvested at different culture times and the expression levels of MAML1 and muscle-specific genes were determined by Western blot analyses.

As shown in Figure 4A, transfection with Maml1 RNAi V and RNAi VII resulted in $>90 \%$ and $80 \%$ decrease, respectively, in endogenous MAML1 protein expression compared with controls. Knockdown of MAML1 expression decreased subsequent myosin expression and myotube formation compared with controls when the cells were induced to differentiate (Fig. 4A,B). Interestingly, this occurred despite the observation that the expression of MAML1 protein appeared to return to nearly normal levels within 2 and $4 \mathrm{~d}$ of culture. In addition, the induction of MyoD and myogenin expression was delayed in the Maml1 RNAi-treated cells (Fig. 4A). In fact, the level of MyoD expression was significantly lower after Maml1 RNAi treatment, suggesting that MAML1 may regulate MyoD expression. Overall, these data demonstrated that MAML1 is required for the induction of muscle-specific genes and the normal differentiation of myoblasts into myotubes in the $\mathrm{C} 2 \mathrm{C} 12$ model.

\section{MAML1 enhances MEF2-mediated transcription}

We next investigated the molecular mechanisms by which MAML1 functions in myogenesis. Since MAML1 functions as a transcriptional coactivator for the Notch signaling pathway (Wu et al. 2000, 2004), we hypothesized that MAML1 may also act as a coactivator for muscle-specific transcription factors. Because expression of exogenous MAML1 up-regulated the MCK promoter reporter, which contains MyoD and MEF2-binding sites, we asked whether MAML1 also coactivates MyoD or MEF2-mediated transcription. First, to determine whether MAML1 is a coactivator for MyoD, C2C12 cells were cotransfected with increasing amounts of MAML1 and MyoD, and the activity of the MCK promoter reporter was measured. As shown in Figure 5A, we found individual expression of exogenous MAML1 or MyoD up-regulated the MCK promoter reporter, but coexpression of MAML1 and MyoD did not have any cooperative effects. These data indicate that MAML1 is unable to function as a coactivator for MyoD.

Next, we examined whether MAML1 coactivates MEF2-mediated transcription. Here, C2C12 cells were cotransfected with an artificial MEF2 reporter (contain-

\section{A}

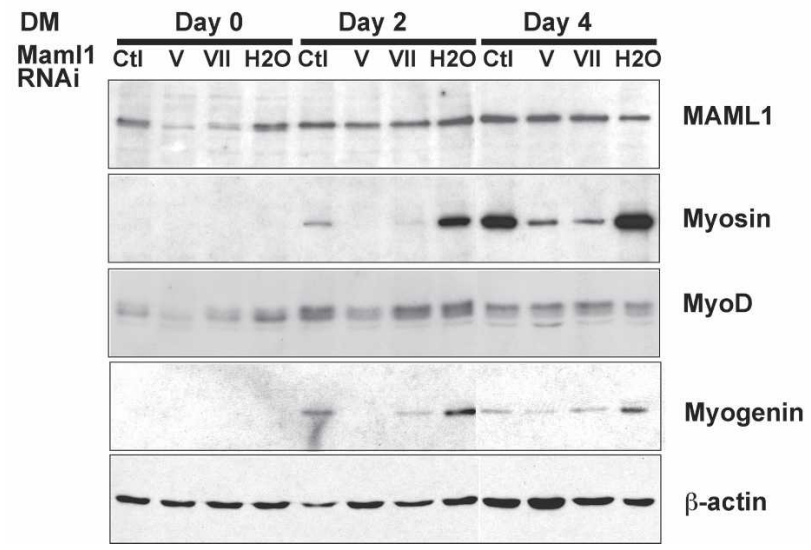

B

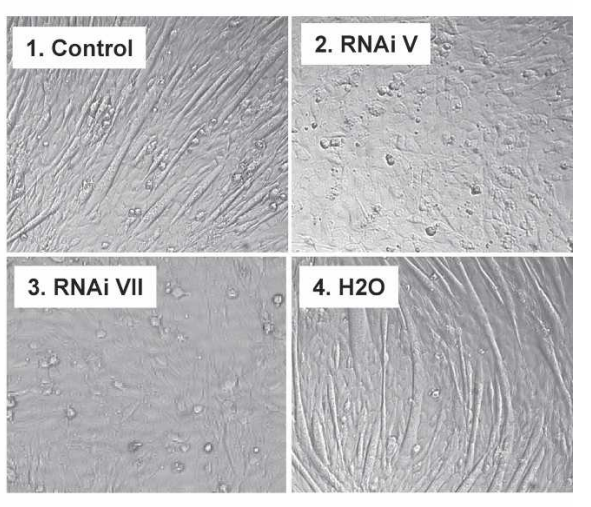

Figure 4. RNAi-mediated knockdown of Maml1 expression inhibits myogenesis in C2C12 cells. (A) RNAi-mediated Maml1 knockdown results in decreased expression of muscle proteins in $\mathrm{C} 2 \mathrm{C} 12$ cells. C2C12 were transfected with two Maml1-specific siRNAs (V and VII), control siRNAs (Ctl), and water on two consecutive days, and cultured in DM at $48 \mathrm{~h}$ after the first transfection for 0, 2, or $4 \mathrm{~d}$. Lysates were harvested for Western blotting. Note that MAML1 expression (shown at top, the upper band) is significantly $(>80 \%-90 \%)$ decreased at day 0 , but then returns to normal levels by day 2 . However, myosin levels are significantly decreased in the cells, while myogenin and MyoD levels appear reduced as well. (B) Phase microscopy reveals that C2C12 cells with knocked down levels of MAML1 fail to form myotubes (panels 2,3), while cells transfected with control siRNAs or water (panels 1,4) form large, mature myotubes within $4 \mathrm{~d}$ of culture. 
A

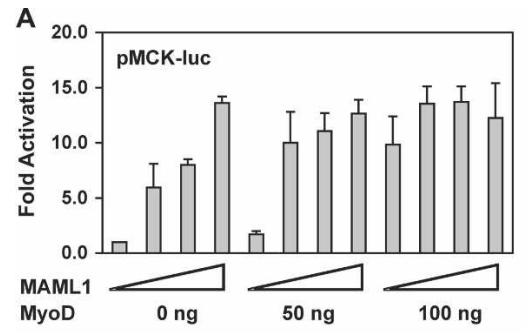

B

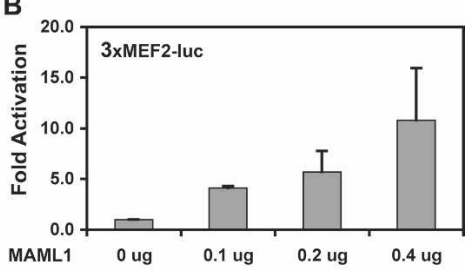

Figure 5. MAML1 and MEF2C act synergistically to activate a MEF2-dependent reporter. (A) MAML1 is not a coactivator for MyoD. C2C12 cells were transfected with $10 \mathrm{ng}$ of Renilla luciferase plasmid, $0.5 \mu \mathrm{g}$ of pMCK-luc reporter, and increasing amounts of expression plasmids encoding Flagtagged MAML1 and/or MyoD. After $24 \mathrm{~h}$ transfection, cells were switched to DM, cultured overnight, and then lysed to prepare cell extracts for luciferase assay. The pMCK-luc reporter activities were expressed as fold activation relative to cells not expressing MAML1 and MyoD. (B) MAML1 activates a MEF2-responsive promoter in a dose-dependent manner. Assays were performed as described above, except the 3xMEF2-luc reporter is used. (C) MAML1 cooperates with MEF2C to activate MEF2-responsive promoter. Assays were performed as described above, except various amount of MEF2C expression vectors were cotransfected with MAML1.

ing three copies of MEF2-responsive elements: 3xMEF2), increasing amounts of MAML1 and/or MEF2 (including MEF2A, MEF2C, or MEF2D) constructs. Indeed, we found that MAML1 activated the MEF2 reporter in a dose-dependent fashion (Fig. 5B), and also cooperated with MEF2C in the reporter activation (Fig. 5C). MAML1's coregulatory function is apparently specific for MEF2C, as we did not observe any cooperative effects between MAML1 and other MEF2 members, MEF2A or MEF2D (Supplementary Fig. S2). The specific, synergistic effect between MEF2C and MAML1 on the MEF2responsive promoter raised the possibility that MAML1 and MEF2C interact.

\section{MEF2C and MAML1 interact in vivo}

To determine whether MAML1 and MEF2C indeed interact, we first performed colocalization studies. We expressed constructs encoding GFP-tagged MEF2C and Flag-tagged MAML1 in U20S osteosarcoma cells. We found that GFP-tagged MEF2C is localized diffusely in the nucleus when expressed ectopically and alone (Fig. 6A, top). However, when coexpressed with MAML1, GFP-MEF2C redistributed into a nuclear dot pattern and colocalized with MAML1 (Fig. 6A, bottom). Similar staining patterns were found when $\mathrm{C} 2 \mathrm{C} 12$ cells were used (data not shown). These data are consistent with MAML1 previously reported in nuclear dots $(\mathrm{Wu}$ et al. 2000), In contrast, we found that MAML1 does not colocalize with another MEF2 member, MEF2A (Supplementary Fig. S3). Therefore, our data support the idea that MAML1 and MEF2C specifically interact.

We also performed Western blot assays on lysates from C2C12 cells coexpressing MAML1 and MEF2C. Interestingly, we found that their coexpression caused a mobility shift of MEF2C (Fig. 6B), likely because MAML1 induced a post-translational modification of MEF2C. The mobility shift was reduced by incubation of the MEF2C immunoprecipitates with calf intestinal alkaline phosphatase (data not shown), suggesting that the modification of MEF2C was due to phosphorylation.

To confirm the in vivo binding of MEF2C and MAML1 and map their respective binding sites, we performed mammalian two-hybrid assays. FL or truncated MEF2C constructs were expressed as fusion proteins with a GAL4 DNA-binding domain (DB) (see Fig. 6C for schematic of constructs), and FL MAML1 was expressed as a fusion protein with the activation domain (AD) in C2C12 cells. The interaction of MEF2C and MAML1 was quantified by the activation of a luciferase reporter containing GAL4-binding sites in the promoter. Our results confirmed that MEF2C and MAML1 interact in vivo; the luciferase activity from cells expressing both proteins was increased $\sim 150$-fold compared with cells expressing either protein plus empty vector. Furthermore, the MEF2C mutant with the deletion of its MADS and MEF domains still retained its ability to bind to MAML1. In addition, the $1-177$ amino acids of the $\mathrm{N}$ terminal region of MEF2C was sufficient to bind to MAML1 (Fig. 6C). Thus, the binding site for MAML1 is located within the 87-177-amino-acid region of the MEF2C protein.

Using similar assays with a series of deletion mutants of MAML1 (Fig. 6D), we found that MEF2C failed to interact with the MAML1 mutant missing its N-terminal 123 amino acids (MAML1 124-1016), but was still able to bind to the mutant missing the internal 71-300 amino acids. These data show that MEF2C interacts within the 1-70-amino-acid region of MAML1. The important function of this MAML1 $\mathrm{N}$-terminal region is supported by our earlier studies, revealing that it is essential for activating muscle-specific gene expression in C2C12 cells (Fig. 3C-E). It should be noted that the interaction between MAML1 and MEF2C might be relatively weak and possibly indirect, as we were not able to 

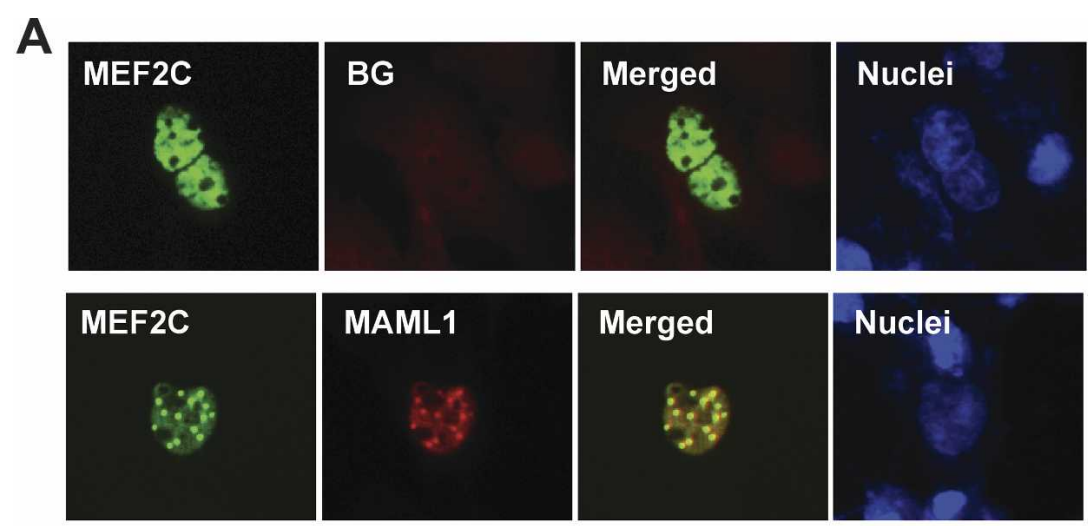

C
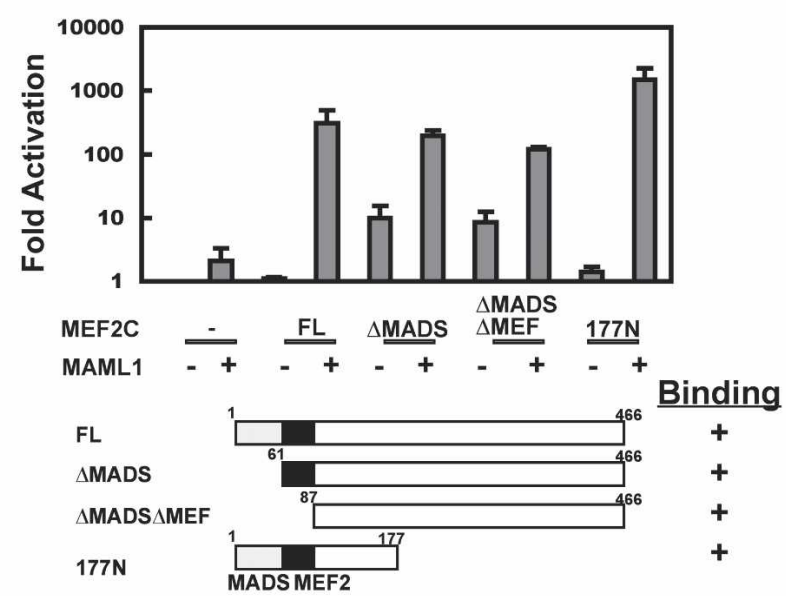

B

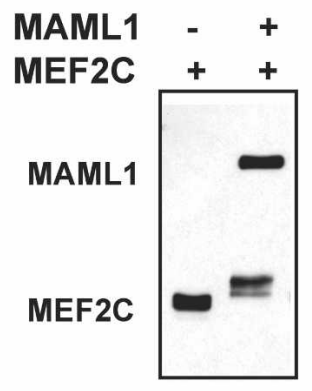

Figure 6. MEF2C and MAML1 interact in vivo. (A) MEF2C and MAML1 colocalize in the nucleus. U20S cells were transfected with the GFP-tagged MEF2C alone (top) or with Flag-tagged MAML1 (bottom), and stained with an anti-Flag antibody to detect MAML1 expression. (BG) Background staining. DAPI staining was performed to label the nuclei. Upon coexpression, MEF2C changed its localization from a diffuse nuclear pattern to colocalize with MAML1 in nuclear dots (merged image at bottom). (B) Coexpression of MAML1 and MEF2C results in post-translational modification of MEF2C. C2C12 cells were transfected with Flag-tagged MEF2C, with or without cotransfection of Flag-tagged MAML1. The expression of both MAML1 and MEF2C were detected by Western blot analysis with anti-Flag antibodies. Note that when the two proteins are coexpressed, the mobility of the MEF2C band shifts. (C) MAML1 interacts with the 87-177-amino-acid region of MEF2C by mammalian two-hybrid assays. C2C12 cells were cotransfected with a firefly luciferase reporter containing GAL4-binding sites (pSG5-luc) along with two expression vectors; i.e., one encoding DB or DB fused to FL or truncated MEF2C, and the other encoding AD or AD fused to MAML1. A total of $0.5 \mu$ g of each construct was used for transfection. Cellular lysates were harvested $44 \mathrm{~h}$ post-transfection. Firefly luciferase activity, normalized with Renilla luciferase expressed from the DB vector, was expressed as fold activation relative to the background level of firefly luciferase expression in the presence of empty $\mathrm{DB}$ and $\mathrm{AD}$ vectors. $(D)$ MEF2C interacts with the N-terminal region of the MAML1, 1-70 amino acids by mammalian two-hybrid assays. Similar assays were carried out as described. FL MEF2C was expressed as DB fusion, while FL or truncated MAML1 expressed as AD fusion.

detect a large amount of MEF2C in MAML1 immunoprecipitates (data not shown). Nonetheless, our results indicate a potentially important interaction between MEF2C and MAML1, which is supported by changes in the nuclear localization and post-translational modification of MEF2C upon coexpression with MAML1, confirmation and mapping of the interaction sites by mammalian two-hybrid assays, and their cooperative activation of a MEF2-responsive promoter. Thus, MAML1 likely coactivates MEF2-mediated gene transcription via its $\mathrm{N}$ terminal region, the same domain previously found to interact with Notch (Wu et al. 2000).

\section{Activation of Notch abrogates MAML1-enhanced myogenesis}

Next, we sought to investigate MAML1 function in the context of Notch signaling during myogenesis. MAML1 is a known component of the active Notch transcriptional complex, and is required for Notch-induced transcriptional activation of downstream targets. It was shown previously that activation of Notch signaling inhibits myogenesis in C2C12 cells (Kopan et al. 1994; Lindsell et al. 1995). However, our data reveal that MAML1 expression enhances myogenesis, while its ab- 
sence inhibits development, and that it functions as a coactivator for MEF2C. Therefore, we designed experiments to examine how MAML1 affects muscle differentiation once the Notch signaling pathway is activated.

C2C12 cells stably transduced with MAML1, or with empty vector, were incubated in culture wells coated with IgG (as a negative control) or the Notch ligand, Delta-Extra-IgG (an IgG fusion with the extracellular domain of Delta ligand), and allowed to differentiate for $6 \mathrm{~d}$. Delta-Extra-IgG, when immobilized on culture wells, activates Notch signaling and blocks differentiation in C2C12 myoblasts (Varnum-Finney et al. 2000). We found that myoblasts overexpressing MAML1 and cultured in control IgG-coated wells differentiated into large, robust myotubes compared with myoblasts transduced with vector alone (Fig. 7A, top). These data are consistent with our previous findings that MAML1 enhances myogenesis (as in Fig. 3C). However, when cultured with

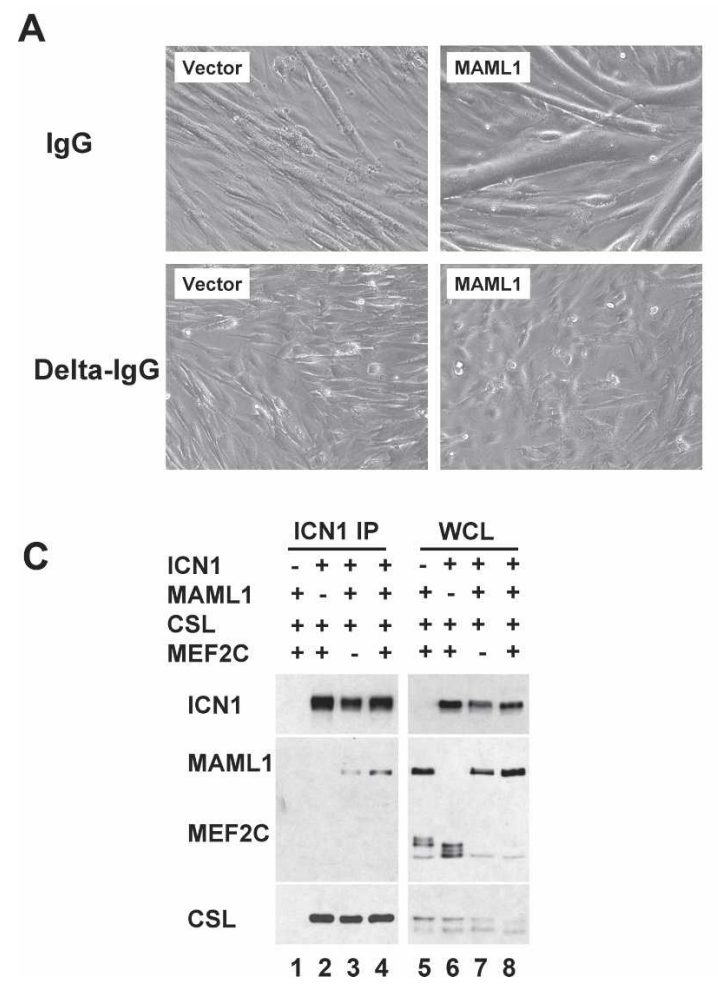

immobilized Delta ligand, both control and MAML1overexpressing myoblasts exhibited a dramatic, morphological block in differentiation (Fig. 7A, bottom). In agreement with this observation, the expression of the constitutively activated form of Notch1, ICN1, inhibited the MAML1-mediated activation of the MCK promoter in $\mathrm{C} 2 \mathrm{C} 12$ cells in a dose-dependent manner (Fig. 7B). Thus, activation of the Notch signaling pathway abrogated MAML1-enhanced myogenesis.

\section{Activation of Notch signaling recruits MAML1 to the Notch transcriptional complex and inhibits Mamll-enhanced MEF2C-dependent transcription}

To investigate the possible mechanisms by which Notch activation overrides MAML1-enhanced myogenesis, we aimed to assess the interactions of MEF2C, MAML1, and Notch ICN1 in the C2C12 myoblasts. By transfecting $\mathrm{C} 2 \mathrm{C} 12$ cells with various combinations of these expres-

B
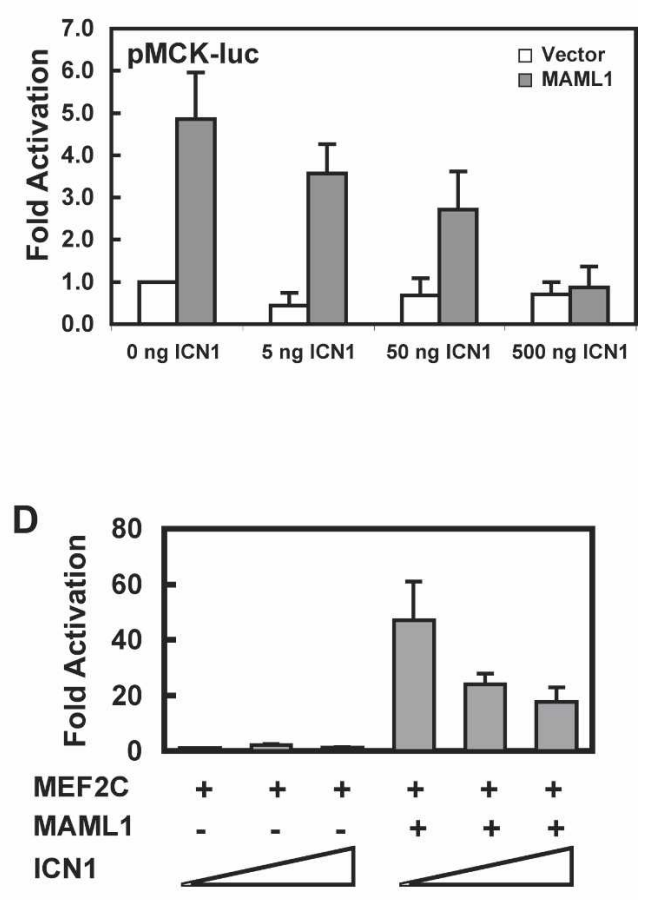

Figure 7. Activation of the Notch signaling pathway overrides MAML1-enhanced myogenesis. (A) Notch ligand stimulation abrogated MAML1-mediated enhancement of myotube formation. C2C12 cells stably transduced with pLXSN based MAML1 viruses or vector control viruses were plated on culture wells coated with either control IgG or ligand Delta-Extra-IgG, and cultured in DM for $6 \mathrm{~d}$. (B) Expression of a constitutively activated form of Notch1 (ICN1) inhibited MAML1-induced activation of MCK promoter. MAML1-transduced C2C12 cells or controls were transfected with $0.5 \mu \mathrm{g}$ of pMCK-luc, $10 \mathrm{ng}$ of Renilla luciferase plasmid, and various amounts of Notch ICN1, and luciferase assays were performed at 44-48 h after transfection. (C) Notch ICN1-MAML1-CSL complex formed in $\mathrm{C} 2 \mathrm{C} 12$ cells. C2C12 cells were cotransfected with different combinations of expression plasmids encoding Flag-tagged MAML1, HA-tagged ICN1, Myc-tagged CSL, and Flag-tagged MEF2C as indicated. Whole-cell lysates (WCL) or anti-HA immunoprecipitates (IP) were blotted with anti-Flag or anti-HA, or anti-Myc antibodies. (D) Expression of ICN1 interfered with the coregulatory function of Maml1 on MEF2C-mediated transcription. C2C12 cells were transfected with $0.5 \mu \mathrm{g}$ of a firefly luciferase reporter containing GAL4-binding sites (pSG5-luc), $0.5 \mu \mathrm{g}$ of the plasmid encoding DB fused to MEF2C, and $0.5 \mu \mathrm{g}$ of expression construct expressing Flag-tagged MAML1 in the presence of various amounts of HA-tagged ICN1 expression constructs $(0,0.25$, and $0.5 \mu \mathrm{g})$. pSG5-luc firefly luciferase activity, corrected for Renilla luciferase activity, is expressed as fold activation relative to cells not expressing MAML1 and ICN1. 
sion plasmids, we detected MAML1 and the Notch-specific transcription factor, CSL, in Notch ICN1 immunoprecipitates (Fig. 7C, lanes 3,4), indicating the formation of the MAML1-ICN1-CSL complex in the C2C12 myoblasts; this complex previously was determined to be essential for the transcription of Notch target genes (Wu et al. 2000). Previously, it was reported that the Notch ICN1 interacts with MEF2C, leading to the inhibition of MEF2C DNA-binding activity (Wilson-Rawls et al. 1999|, suggesting that this is one of the CSL-independent mechanisms for Notch-induced inhibition of myogenesis. However, we could not detect MEF2C in the ICN1 immunoprecipates under the conditions we used (Fig. 7C, lane 2), indicating that the Notch ICN1-MAML1CSL complex may be more stable than the ICN1-MEF2C complex. These data suggest that CSL-dependent mechanisms-i.e., transcription of Notch target genes-may be one of the major mechanisms for Notch effects. Interestingly, we found that expression of the combination of MAML1 and ICN1, but not individually, decreased exogenous MEF2C expression (Fig. 7C, cf. lanes 5,6,8), suggesting that MEF2C expression might be regulated by both MAML1 and ICN1. Overall, our data suggest that MAML1 might predominantly function as a coactivator for Notch receptors instead of for MEF2C when Notch signaling is activated. Indeed, we found that expression of ICN1 inhibits MAML1-enhanced MEF2C-mediated transcription in a dose-dependent fashion in luciferase assays, indicating that MAML1's coactivator function for MEF2C is greatly reduced in the context of Notch activation (Fig. 7D).

Our studies thus reveal that once the Notch pathway is activated, MAML1 is recruited from its role as a coactivator for MEF2-mediated transcription to that of a Notch transcriptional coactivator. The consequence of this role switching of MAML1 is expected to result in an expression of Notch targets and an inhibition of myogenesis once the Notch signaling pathway is activated.

\section{Discussion}

Transcriptional coactivators are proteins that, along with transcription factors, exist in multiprotein complexes and mediate transcriptional events. However, coactivators are not solely components of the basic transcriptional machinery, since recent studies implicate them as primary targets of developmental and physiological signals in many diverse biological processes (Spiegelman and Heinrich 2004). Our present study reveals a novel role for MAML1, a known coactivator for Notch. Mice with a targeted deletion of the Maml1 gene were found to have dystrophic skeletal muscles, and embryonic fibroblasts from these mice failed to undergo muscle differentiation in vitro. Interestingly, exogenous MAML1 expression in C2C12 cells promoted significantly enhanced myotube formation and muscle gene expression, while RNAi-mediated knockdown of MAML1 abrogated muscle differentiation, suggesting that at least some of the effects of MAML1 on muscle development could be Notch independent. In support of this notion, MAML1 was found to bind to MEF2C and to function as a potent coactivator. However, activated Notch blocked the myogenic effects of MAML1 and MEF2C, possibly by recruiting MAML1 into the Notch transcriptional complex. Thus, MAML1 is essential for proper muscle development and may function both to promote and inhibit muscle differentiation, depending on context.

\section{Maml1 is essential for myogenic differentiation in vivo and in vitro}

Maml1 is one of three mammalian homologs of Drosophila mastermind, a gene that was genetically linked to the Notch signaling pathway in flies (Xu et al. 1990). Previously, biochemical studies determined that all three mammalian MAML proteins exhibited differential transcriptional coactivities for different Notch receptors (Wu et al. 2002), and some MAML family members have been implicated in cancer (Tonon et al. 2003). In addition, a dominant-negative mutant of MAML1 interfered with Notch-mediated T/B-cell fate decisions in mouse bone marrow (Maillard et al. 2004), indicating an important role for MAML1 in mediating Notch signaling. However, the exact in vivo roles of the MAML family of transcriptional coactivators have not been established.

Here, we generated a Maml1 KO mouse and found that an absence of Maml1 results in death within the perinatal period. The exact cause(s) of death are currently unknown and being investigated. Strikingly, the Maml1null mice exhibit severe skeletal muscle defects typical of a muscular dystrophy. While defective Notch signaling could contribute to abnormal muscle development, we found that MEFs from Maml1-null mice failed to undergo MyoD-induced myogenic differentiation in vitro, suggesting that there were intrinsic defects in muscle differentiation. Indeed, our data using C2C12 cells further indicate that the muscular dystrophy in the Maml1-null mice is most likely a result of a severe perturbation in myogenesis, with a disruption in key interactions between MAML1-MEF2C- and MAML1-Notchmediated pathways. However, it must be noted that, although the Maml1-null muscles are severely perturbed in their structure, they are "functional" enough to allow the mice to live beyond the embryonic stage. This may be due to expression of the two other members of the Maml family, Maml2 and Maml3, also found expressed in human skeletal muscles (Wu et al. 2002), or a result of compensatory effects from these genes. Future studies on the expression and functional properties of these two members will help in understanding the muscle defects in the KO mice, and the potential roles of other $M A M L$ family members in muscle development.

\section{MAML1 coactivates MEF2C to promote the transcription of muscle-specific genes}

MEF2C is one of four MEF2 gene family members in mammals encoding key transcription factors that medi- 
ate gene expression in myocytes (Black and Olson 1998). Mice lacking Mef2c die at E9.5 due to defects in heart looping (Lin et al. 1997), and MEF2C is also known to regulate skeletal muscle-specific genes including $\mathrm{MCK}$, desmin, and myogenin. One recent study showed that the loss of SrpK3 kinase, one of the MEF2C downstream targets, resulted in centronuclear myopathy in mice (Nakagawa et al. 2005). In addition, MEF2C is able to interact with MRFs to potentiate their transcriptional activities.

Previously, it was reported that a number of coactivators including GRIP1 (Chen et al. 2000), CARM1 (Chen et al. 2002), PGC1 (Michael et al. 2001), and p300 (Sartorelli et al. 1997) enhance MEF2C-dependent transcription. In this regard, diverse signaling pathways regulate MEF2C activities by interfering with its interactions with coactivators, some of which are known to perturb the myogenic program. For instance, cyclin D-cdc4 kinase prevents the activation of MEF2C by blocking its interaction with the coactivator GRIP1, thereby suppressing the muscle differentiation program in proliferating myoblasts (Lazaro et al. 2002). Transforming growth factor $\beta$ (TGF- $\beta$ ) suppresses myogenic differentiation via its effector, Smad3, which is in part capable of disrupting MEF2C and GRIP1 association (Liu et al. 2004). This evidence suggests a critical, functional significance in MEF2C-mediated transcription in myogenesis. Indeed, here we identified another mechanism by which MEF2C is regulated in myogenesis, via its coactivator, MAML1.

The respective interaction domains of MAML1 and MEF2C were mapped to the N-terminal domain of the MAML1 (which also interacts with Notch receptors), and the region just adjacent to the MADS-MEF2 domains of MEF2C (a binding site that differs from the reported GRIP1-binding site) by mammalian two-hybrid assays. It should be noted that the interaction of MAML1 and MEF2C might be relatively weak based on our coimmunoprecipitation studies, and it is unclear whether such interaction is direct in vivo. Nevertheless, MAML1 and MEF2C interaction sufficiently activates MEF2-mediated promoters. We found that MAML1 is a potent coactivator of MEF2C, especially in comparison with one of the MEF2C's previously described coactivators, GRIP1 (data not shown). Combining our data with other studies, we can propose at least two mechanisms by which MAML1 is able to coactivate MEF2C transcriptional events. First, we found that in our overexpression studies, the MAML1-MEF2C interaction resulted in post-translational modifications of MEF2C, likely phosphorylation, which could contribute to MAML1-mediated potentiation of MEF2C transcription. Consistently with this observation, MAP kinases, both p38 and extracellular signal-regulated kinase (ERK), enhance the transcriptional activities of MEF2C via phosphorylation (Yang et al. 1999). Since the endogenous levels of MAML1 in the cells are low, it remains unclear regarding the amount of physiological MEF2C that is phosphorylated by endogenous MAML1 and the functional impact of such modification. Thus, further studies in- vestigating potential phosphorylation sites, the responsible kinase(s), and whether phosphorylation regulates the DNA-binding and/or transcriptional activities of MEF2C, are warranted. Second, based on mammalian two-hybrid assays, MAML1 and MEF2C physically interact, bringing additional transcriptional regulators to MEF2C through MAML1, including p300 (Fryer et al. 2002) and likely other currently unknown proteins.

Finally, an important future question is to understand exactly how the MEF2C and MAML1 interaction regulates the myogenic program, and the exact target genes that are controlled by the complex. In this regard, we found that RNAi-mediated down-regulation of MAML1 expression, as well as the expression of a $\mathrm{N}$-terminal truncated mutant of MAML1 in C2C12 myoblasts, both resulted in a delayed induction of the key myogenic regulators, MyoD, and myogenin. Since a key proximal MEF2 site in the mouse myogenin promoter regulates myogenin expression levels (Cheng et al. 1993), it is likely that the loss of MAML1/MEF2C interaction accounts for the delayed induction of myogenin in Maml1siRNA-treated cells. The absence of the MAML1MEF2C interaction in Maml1-null mice may lead to decreased myogenin expression, contributing to muscle defects.

\section{Maml1 is involved in cross-stalk between Notch signaling and MEF2C signaling}

We initially speculated that MAML1, as an essential coactivator for Notch signaling, might contribute to the Notch-mediated inhibition of myogenesis. Surprisingly, however, we discovered a novel promyogenic differentiation function for the Maml1 gene. These seeming paradoxical results prompted us to examine how MAML1 affects muscle differentiation in the context of Notch activation. In fact, upon Notch activation, the MAML1mediated enhancement of myogenesis was abrogated. Therefore, it appears that MAML1's role of a coactivator for the Notch signaling pathway dominates over its role in coactivating MEF2C-mediated muscle gene transcription. Mechanistically, Notch activation led to a recruitment of MAML1 away from MEF2C to the Notch/CSL transcriptional complex.

Taken together, our data allow us to propose a model for the role of MAML1 in myogenesis that may at least partially explain the Notch-induced inhibition of myogenesis. (1) In the absence of the activated Notch pathway (i.e., during events that occur after myoblast proliferation and recruitment), MAML1 serves as a transcriptional coactivator for the muscle-specific transcription factor MEF2C. Together, they regulate muscle gene expression, leading to enhanced myogenic events. (2) Upon activation of Notch receptors (e.g., induction of Notch ligand delta after muscle injury) MAML1 switches its role from a transcriptional coactivator of MEF2C to that of a transcriptional coactivator for Notch-CSL, resulting in the expression of specific Notch target genes. Thus, MAML1 plays dual roles as transcriptional coactivators for muscle-specific genes as well as for target genes for 
the Notch pathway. The "functional switching" of MAML1 in response to signals to activate Notch receptors likely contributes to the CSL-dependent inhibition of myogenesis.

Therefore, with our current knowledge about the regulated role of Notch in different stages of myogenesis, the loss of MAML1 expression in the Maml1 KO mice may have both Notch-dependent and Notch-independent myogenic defects that contribute to muscular dystrophy. For example, the loss of the MAML1-Notch interaction may result in decreased Notch signaling that is required for proliferation and expansion of muscle stem cells, and thus affect muscle regeneration. Currently, the extent of Notch signaling affected in the mutant muscles is still unknown, because Notch targets in the muscles are not well defined. However, we found that the expression levels of a Notch target gene, HES1, seemed to be slightly decreased in the postnatal day 1 (P1) hind-limb muscles of the maml1 KO mice as compared with the wild-type mice (Supplementary Fig. S4). On the other hand, the loss of MAML1-MEF2C might lead to impaired myogenic differentiation. Future research is required to assess the functional contributions of these two potential aspects of MAML1 functions in understanding the muscular dystrophy in Maml1 KO mice.

Finally, aspects of the model proposed in this study may contribute to understanding how the Notch signaling pathway regulates multiple biological processes, with very different cellular outcomes. Our previous studies suggest that the MAML family, with its differential expression patterns and distinct interactions with Notch receptors, could mediate Notch pathway effects via coactivation of transcription. The present study suggests the exciting possibility that the MAML family serves as coactivators for other transcription factors and mediates cross-talks between Notch and different biological signaling pathways.

\section{Materials and methods}

\section{Generation of Maml1 KO mice}

The gene structure of murine Maml1 has been previously described (Wu et al. 2004). Screening of a BAC Mouse II Hybridization library was performed (Incyte Genomics, Inc.) using a 324-bp probe derived from the $5^{\prime}$ end of the murine Maml1 cDNA (encoding 1-97 amino acids). One positive clone contained the entire genomic sequence for the Maml1 gene. A Maml1 targeting vector was subsequently made with the Neo gene replacing the $5^{\prime}$ promoter region and exon 1 . The vector was linearized with NotI and electroporated into J129 mouse embryonic stem (ES) cells. ES cell clones that survived repeated selection were chosen for Southern blot analyses. The genomic probe used for Southern blotting was external to the genomic DNA used in targeting vector, and XmnI digest yielded a 11.7$\mathrm{kb}$ band in the wild-type allele and a $23.3-\mathrm{kb}$ band in the targeted allele. Two of the ES clones containing the correctly targeted allele were individually injected into C57BL/6 blastocysts, one of which gave rise to the chimeric mice that transmit the targeted allele to the germline. PCR genotyping of mice was performed with the primers for the Neo gene (Top, 5'-ATT

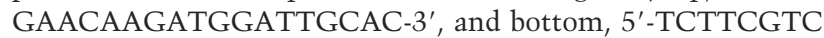

CAGATCATCCT- $3^{\prime}$ ) to detect the targeted allele, and with the primers for the deleted region of the Maml1 (top, 5'-GC CACTCCCGCCACCAAAAAC-3', and bottom, 5'-TTTCC GACCTCATTCTTTACA-3') to detect the wild-type allele.

\section{Histology}

For routine histological analysis, embryos or tissue samples were fixed in Bouin's solution and embedded in paraffin for sectioning. Tissue sections were stained with H\&E. These procedures were performed at the Rodent Histopathology Core at Harvard Medical School.

\section{Establishment of wild-type and Maml1 KO embryonic fibroblast cell lines, and myogenesis assay}

Primary wild-type and Maml1 KO MEFs were isolated from E12.5 littermate embryos and cultured in DMEM plus 10\% fetal bovine serum (FBS). Early passage (less than five) MEFs were plated at a density of $2.7 \times 10^{6}$ cells per $10-\mathrm{cm}$ dish, and replated at the same density every $3 \mathrm{~d}$ (Todaro and Green 1963). Cells were immortalized after 20-30 passages. For myogenic conversion assays, MEFs were infected with pMSCV-based MyoD and/ or pLXSN-based MAML1 viruses, and selected in growth medium (DMEM plus $20 \%$ FBS) with $2 \mu \mathrm{g} / \mathrm{mL}$ of puromycin or 250 $\mu \mathrm{g} / \mathrm{mL}$ of G418. Cells were grown to $80 \%-90 \%$ confluence and switched to differentiation medium (DMEM containing 2\% horse serum and $10 \mu \mathrm{g} / \mathrm{mL}$ insulin) for $3 \mathrm{~d}$.

\section{Cell culture, retroviral transduction, transfection, and differentiation assay}

C2C12 cells (ATCC) were cultured in DMEM with 20\% inactivated fetal calf serum (FCS). U2OS cells were cultured in DMEM with $10 \%$ FCS. Cells were infected with pLXSN-based retroviruses, and selected with G418 at $250 \mu \mathrm{g} / \mathrm{mL}$. Transfections with Maml1 siRNA (to a final concentration of $100 \mathrm{nM}$ ) were carried out in the six-well plates using Lipofectamine Plus Reagent (Invitrogen) for two consecutive days. Two Maml1-specific siGenome duplexes V (D-059179-05) and VII (D-059179$07)$, and control nontargeting siRNA pool were ordered from Dharmarcon. For the myogenesis assay, C2C12 cells were grown to $80 \%-90 \%$ confluence, and induced for differentiation by switching from growth medium to differentiation medium (DM: DMEM containing 2\% horse serum).

\section{Northern and Western blot analyses}

Total RNA isolated by TRIZOL reagent (Invitrogen) was subjected to Northern blot analysis as described previously (Wu et al. 2000). Whole-cell protein extracts were prepared for Western blot and immunoprecipitation studies as described previously (Wu et al. 2000). The following antibodies were used: the late muscle marker, myosin heavy chain (Clone MY32), Flag (M2), Myc (9E10), and $\beta$-actin from Sigma; Myogenin and MyoD (Clone 5.8A) from PharMingen; HA from BabCo; and anti-HA affinity matrix from Roche. Rabbit Maml1 antibodies (BL1237 and BL1239) were provided by Bethyl Laboratories, Inc.

\section{Plasmids}

FL MAML1 and two truncated mutants, MAML1(1-302) and MAML1(124-1016), were cloned into the pLXSN vector (Clontech). MAML1(303-1016) and MAML1(D71-300) were cloned into the pFlag-CMV2 vectors (Sigma). MEF2C was recloned from pCMX-MEF2C-Flag plasmid (Lazaro et al. 2002) to the 
pEGFP C3 vector (Clontech) to express GFP-MEF2C fusion. FL MEF2C, $\triangle$ MADS containing MEF2C 61-466 amino acids, $\triangle$ MADS $\triangle$ MEF containing MEF2C 87-466 amino acids, and $177 \mathrm{~N}$ containing MEF2C 1-177 amino acids were cloned into pBIND vector (Promega). 3xMEF2-luc containing three copies of MEF2-responsive sites in pGL3 vector and MEF2A, MEF2B, and MEF2C in pcDNA vector were obtained from Eric Olson (University of Texas Southwestern Medical Center at Dallas, Dallas, TX); MyoD in pMSCV puro vector were from Robert Roeder (The Rockefeller University, New York, NY); HA-tagged HA-p300 in pCMV were from David Livingston (Dana-Farber Cancer Institute, Boston, MA); HA-tagged GRIP1 in pSG5 vector were from Michael Stallcup (University of Southern California, Los Angeles, CA); and pMCK-luc were from Andrew Lassar (Harvard Medical School, Boston, MA). FL MAML1, MAML1(1-302), and MAML1(124-1016) in pFlag-CMV2; Myctagged CSL and HA-tagged ICN1 in pcDNA vector; and pSG5luc (Promega) have all been described previously (Wu et al. 2002).

\section{Reporter assays}

$\mathrm{C} 2 \mathrm{C} 12$ cells were cultured at $1 \times 10^{5}$ cells/well in six-well plates and transfected the following day with cDNA constructs as indicated in the figure legends using Superfect Transfection reagent (Qiagen). Luciferase-based reporter assays were performed as described previously (Wu et al. 2000).

\section{Immunofluorescence Staining}

Staining was performed as described previously (Wu et al. 2002).

\section{Acknowledgments}

We thank Irwin Bernstein, Andrew Lassar, David Livingston, Eric Olson, Robert Roeder, and Michael Stallcup for the reagents; Carol Gregorio and Parker Antin for critical reading of our manuscript; David Besselsen for tissue section analysis; and Makoto Nakamura for technical help. This work was supported in part by NIH RO1 CA036167 to J.D.G., NIH RO1 CA097148 to L.W., and an American Heart Association grant 0435316N to A.S.M. H.S. (ZOC) is also supported by Natural Science Foundation of China (30270530 and 30470677) and Guangdong Natural Science Foundation (021825).

\section{References}

Artavanis-Tsakonas, S., Rand, M.D., and Lake, R.J. 1999. Notch signaling: Cell fate control and signal integration in development. Science 284: 770-776.

Bailey, P., Holowacz, T., and Lassar, A.B. 2001. The origin of skeletal muscle stem cells in the embryo and the adult. Curr. Opin. Cell Biol. 13: 679-689.

Berkes, C.A. and Tapscott, S.J. 2005. MyoD and the transcriptional control of myogenesis. Semin. Cell Dev. Biol. 16: 585595.

Black, B.L. and Olson, E.N. 1998. Transcriptional control of muscle development by myocyte enhancer factor-2 (MEF2) proteins. Annu. Rev. Cell Dev. Biol. 14: 167-196.

Chen, S.L., Dowhan, D.H., Hosking, B.M., and Muscat, G.E. 2000. The steroid receptor coactivator, GRIP-1, is necessary for MEF-2C-dependent gene expression and skeletal muscle differentiation. Genes \& Dev. 14: 1209-1228.

Chen, S.L., Loffler, K.A., Chen, D., Stallcup, M.R., and Muscat, G.E. 2002. The coactivator-associated arginine methyltrans- ferase is necessary for muscle differentiation: CARM1 coactivates myocyte enhancer factor-2. J. Biol. Chem. 277: 43244333.

Cheng, T.C., Wallace, M.C., Merlie, J.P., and Olson, E.N. 1993. Separable regulatory elements governing myogenin transcription in mouse embryogenesis. Science 261: 215-218.

Conboy, I.M. and Rando, T.A. 2002. The regulation of Notch signaling controls satellite cell activation and cell fate determination in postnatal myogenesis. Dev. Cell 3: 397-409.

Conboy, I.M., Conboy, M.J., Smythe, G.M., and Rando, T.A. 2003. Notch-mediated restoration of regenerative potential to aged muscle. Science 302: 1575-1577.

Dias, P., Dilling, M., and Houghton, P. 1994. The molecular basis of skeletal muscle differentiation. Semin. Diagn. Pathol. 11: 3-14.

Fryer, C.J., Lamar, E., Turbachova, I., Kintner, C., and Jones, K.A. 2002. Mastermind mediates chromatin-specific transcription and turnover of the Notch enhancer complex. Genes \& Dev. 16: 1397-1411.

Kopan, R., Nye, J.S., and Weintraub, H. 1994. The intracellular domain of mouse Notch: A constitutively activated repressor of myogenesis directed at the basic helix-loop-helix region of MyoD. Development 120: 2385-2396.

Lazaro, J.B., Bailey, P.J., and Lassar, A.B. 2002. Cyclin D-cdk4 activity modulates the subnuclear localization and interaction of MEF2 with SRC-family coactivators during skeletal muscle differentiation. Genes \& Dev. 16: 1792-1805.

Lin, Q., Schwarz, J., Bucana, C., and Olson, E.N. 1997. Control of mouse cardiac morphogenesis and myogenesis by transcription factor MEF2C. Science 276: 1404-1407.

Lin, S.E., Oyama, T., Nagase, T., Harigaya, K., and Kitagawa, M. 2002. Identification of new human mastermind proteins defines a family that consists of positive regulators for notch signaling. J. Biol. Chem. 277: 50612-50620.

Lindsell, C.E., Shawber, C.J., Boulter, J., and Weinmaster, G. 1995. Jagged: A mammalian ligand that activates Notch1. Cell 80: 909-917.

Liu, D., Kang, J.S., and Derynck, R. 2004. TGF- $\beta$-activated Smad3 represses MEF2-dependent transcription in myogenic differentiation. EMBO J. 23: 1557-1566.

Luo, D., Renault, V.M., and Rando, T.A. 2005. The regulation of Notch signaling in muscle stem cell activation and postnatal myogenesis. Semin. Cell Dev. Biol. 16: 612-622.

Maillard, I., Weng, A.P., Carpenter, A.C., Rodriguez, C.G., Sai, H., Xu, L., Allman, D., Aster, J.C., and Pear, W.S. 2004. Mastermind critically regulates Notch-mediated lymphoid cell fate decisions. Blood 104: 1696-1702.

McKinsey, T.A., Zhang, C.L., and Olson, E.N. 2001. Control of muscle development by dueling HATs and HDACs. Curr. Opin. Genet. Dev. 11: 497-504.

Michael, L.F., Wu, Z., Cheatham, R.B., Puigserver, P., Adelmant, G., Lehman, J.J., Kelly, D.P., and Spiegelman, B.M. 2001. Restoration of insulin-sensitive glucose transporter (GLUT4) gene expression in muscle cells by the transcriptional coactivator PGC-1. Proc. Nat. Acad. Sci. 98: 38203825 .

Molkentin, J.D., Black, B.L., Martin, J.F., and Olson, E.N. 1995. Cooperative activation of muscle gene expression by MEF2 and myogenic bHLH proteins. Cell 83: 1125-1136.

Nakagawa, O., Arnold, M., Nakagawa, M., Hamada, H., Shelton, J.M., Kusano, H., Harris, T.M., Childs, G., Campbell, K.P., Richardson, J.A., et al. 2005. Centronuclear myopathy in mice lacking a novel muscle-specific protein kinase transcriptionally regulated by MEF2. Genes \& Dev. 19: 2066-2077.

Nofziger, D., Miyamoto, A., Lyons, K.M., and Weinmaster, G. 
Shen et al.

1999. Notch signaling imposes two distinct blocks in the differentiation of $\mathrm{C} 2 \mathrm{C} 12$ myoblasts. Development 126: 1689-1702.

Novitch, B.G., Mulligan, G.J., Jacks, T., and Lassar, A.B. 1996. Skeletal muscle cells lacking the retinoblastoma protein display defects in muscle gene expression and accumulate in S and G2 phases of the cell cycle. J. Cell Biol. 135: 441-456.

Parker, M.H., Seale, P., and Rudnicki, M.A. 2003. Looking back to the embryo: Defining transcriptional networks in adult myogenesis. Nat. Rev. Genet. 4: 497-507.

Rida, P.C., Le Minh, N., and Jiang, Y.J. 2004. A Notch feeling of somite segmentation and beyond. Dev. Biol. 265: 2-22.

Sartorelli, V., Huang, J., Hamamori, Y., and Kedes, L. 1997. Molecular mechanisms of myogenic coactivation by p300: Direct interaction with the activation domain of MyoD and with the MADS box of MEF2C. Mol. Cell. Biol. 17: 10101026.

Shawber, C., Nofziger, D., Hsieh, J.J., Lindsell, C., Bogler, O., Hayward, D., and Weinmaster, G. 1996. Notch signaling inhibits muscle cell differentiation through a CBF1-independent pathway. Development 122: 3765-3773.

Spiegelman, B.M. and Heinrich, R. 2004. Biological control through regulated transcriptional coactivators. Cell 119: 157-167.

Todaro, G.J. and Green, H. 1963. Quantitative studies of the growth of mouse embryo cells in culture and their development into established lines. J. Cell Biol. 17: 299-313.

Tonon, G., Modi, S., Wu, L., Kubo, A., Coxon, A.B., Komiya, T., O’Neil, K., Stover, K., El-Naggar, A., Griffin, J.D., et al. 2003. $\mathrm{t}(11 ; 19)(\mathrm{q} 21 ; \mathrm{p} 13)$ translocation in mucoepidermoid carcinoma creates a novel fusion product that disrupts a Notch signaling pathway. Nat. Genet. 33: 208-213.

Varnum-Finney, B., Wu, L., Yu, M., Brashem-Stein, C., Staats, S., Flowers, D., Griffin, J.D., and Bernstein, I.D. 2000. Immobilization of Notch ligand, Delta-1, is required for induction of notch signaling. J. Cell Sci. 23: 4313-4318.

Weinmaster, G. and Kintner, C. 2003. Modulation of notch signaling during somitogenesis. Annu. Rev. Cell Dev. Biol. 19: 367-395.

Wilson-Rawls, J., Molkentin, J.D., Black, B.L., and Olson, E.N. 1999. Activated notch inhibits myogenic activity of the MADS-Box transcription factor myocyte enhancer factor 2C. Mol. Cell. Biol. 19: 2853-2862.

Wu, L., Aster, J.C., Blacklow, S.C., Lake, R., Artavanis-Tsakonas, S., and Griffin, J.D. 2000. MAML1, a human homologue of Drosophila mastermind, is a transcriptional co-activator for NOTCH receptors. Nat. Genet. 26: 484-489.

Wu, L., Sun, T., Kobayashi, K., Gao, P., and Griffin, J.D. 2002. Identification of a family of mastermind-like transcriptional coactivators for mammalian notch receptors. Mol. Cell. Biol. 22: 7688-7700.

Wu, L., Kobayashi, K., Sun, T., Gao, P., Liu, J., Nakamura, M., Weisberg, E., Mukhopadhyay, N.K., and Griffin, J.D. 2004. Cloning and functional characterization of the murine mastermind-like 1 (Maml1) gene. Gene 328: 153-165.

$\mathrm{Xu}, \mathrm{T} .$, Rebay, I., Fleming, R.J., Scottgale, T.N., and ArtavanisTsakonas, S. 1990. The Notch locus and the genetic circuitry involved in early Drosophila neurogenesis. Genes \& Dev. 4: 464-475.

Yang, S.H., Galanis, A., and Sharrocks, A.D. 1999. Targeting of p38 mitogen-activated protein kinases to MEF2 transcription factors. Mol. Cell. Biol. 19: 4028-4038. 


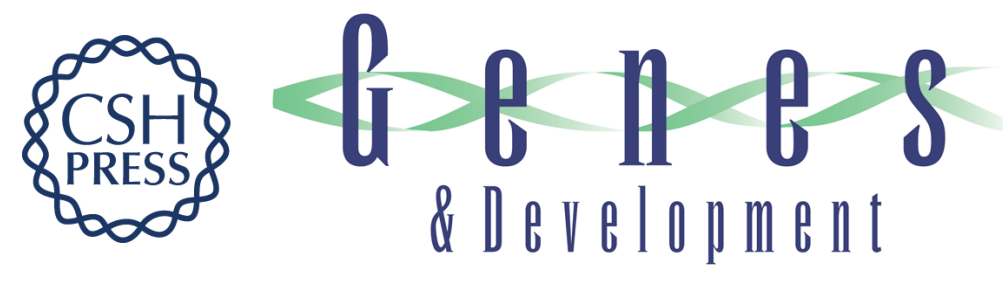

\section{The Notch coactivator, MAML1, functions as a novel coactivator for MEF2C-mediated transcription and is required for normal myogenesis}

Huangxuan Shen, Abigail S. McElhinny, Yang Cao, et al.

Genes Dev. 2006, 20:

Access the most recent version at doi:10.1101/gad.1383706

Supplemental http://genesdev.cshlp.org/content/suppl/2006/03/01/gad.1383706.DC1
Material

References This article cites 40 articles, 21 of which can be accessed free at:

http://genesdev.cshlp.org/content/20/6/675.full.html\#ref-list-1

License

Email Alerting Receive free email alerts when new articles cite this article - sign up in the box at the top

Service

right corner of the article or click here.

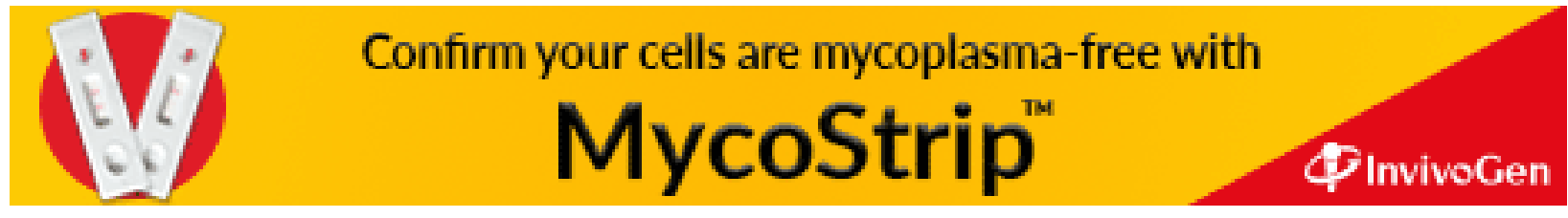

